\title{
La cohérence terminologique en question dans le Manual de gramática francesa de Echeverría Pereda
}

Terminological coherence in question in the Manual de gramática francesa by Echeverría Pereda

\section{Élodie Weber}

\section{OpenEdition}

Édition électronique

URL : https://journals.openedition.org/dhfles/8503

DOI : $10.4000 /$ dhfles.8503

ISSN : 2221-4038

Éditeur

Société Internationale pour l'Histoire du Français Langue Étrangère ou Seconde

Édition imprimée

Date de publication : 1 décembre 2021

ISSN : 0992-7654

\section{Référence électronique}

Élodie Weber, «La cohérence terminologique en question dans le Manual de gramática francesa de Echeverría Pereda », Documents pour l'histoire du français langue étrangère ou seconde [En ligne], 66-67 | 2021, mis en ligne le 02 mars 2022, consulté le 28 mars 2023. URL : http://journals.openedition.org/ dhfles/8503; DOI : https://doi.org/10.4000/dhfles.8503

Ce document a été généré automatiquement le 28 mars 2023.

Tous droits réservés 


\title{
La cohérence terminologique en question dans le Manual de gramática francesa de Echeverría Pereda
}

\author{
Terminological coherence in question in the Manual de gramática francesa by \\ Echeverría Pereda
}

Élodie Weber

\section{Introduction}

Que l'on considère le domaine français ou espagnol, la terminologie grammaticale a toujours souffert, même dans les discours savants, d'un flou que déplorent les linguistes: "À la relative unité de la description du latin a [...] succédé, à la Renaissance, $d u$ fait des emprunts directs, calques ou transpositions dans les langues d'Europe de ses catégorisations, une dispersion dans l'espace et le temps de termes voisins et apparentés » (Beacco $2015:$ 11). Un certain nombre de travaux se sont ainsi intéressés aux origines de la terminologie grammaticale ou au problème des mouvements terminologiques dans les grammaires savantes, à travers la diachronie ${ }^{1}$. Mais le problème de la terminologie ne se pose pas uniquement dans les discours savants; comme le rappelle Beacco (2015: 12 idem), les origines de la terminologie grammaticale et des descriptions qui leur correspondent sont multiples « en ce qu'elles impliquent des spécialistes, 'grammairiens' puis linguistes, des enseignants, des locuteurs natifs, des élèves/apprenants ». Qu'elles soient le fait d'acteurs savants, de grammairiens ou d'enseignants prenant appui sur leur propre expertise, les grammaires dites pédagogiques, dont le but est de rendre accessible à des apprenants non spécialistes les savoirs savants de la grammaire, opèrent une "adaptation ", «transformation cognitive et de reformulation verbale des savoirs/discours savants de la grammaire du français " (Bruley et al. 2012: 10). La simplification ou modification d'une terminologie déjà fluctuante s'avère être l'un des procédés de la transposition 
didactique, décrite par Chevallard (1991:39) comme l'une des manifestations de l'adaptation.

2 Le problème se pose avec une particulière acuité dans le domaine de la didactique des langues et, par conséquent, des grammaires pédagogiques de langue étrangère. Se donnant pour tâche de décrire le fonctionnement d'une langue non encore comprise ni parlée par leurs utilisateurs potentiels, les grammaires de langue étrangère doivent quant à elles « contextualiser » ces savoirs savants en plus de les adapter, c'est-à-dire leur «faire subir une nouvelle transformation [...] de manière à les rendre plus compréhensibles et assimilables en fonction du contexte d'enseignement/ apprentissage » (Bruley et al. 2012 : 11). Le phénomène s'observe en particulier dans les grammaires élaborées par des locuteurs non natifs du français qui, confrontés aux difficultés d'appropriation de leurs élèves, ressentent la nécessité d'adapter le contenu grammatical disponible afin de faciliter l'apprentissage de la langue étrangère : mise en œuvre de l'approche contrastive, modification de la description grammaticale ellemême à travers un ensemble de procédés décrits par Bruley et al. (2012) et Fouillet (2014).

3 Centrale dans l'élaboration d'une grammaire de FLE adressée à un public ayant en commun une même langue maternelle, la question des catégories grammaticales et de la terminologie employée pour les nommer relève à la fois de l'adaptation et de la contextualisation: d'une part la terminologie subira, comme dans toute grammaire pédagogique, un processus de simplification; d'autre part il est fort à parier que le contexte linguistique et la culture métalinguistique des apprenants orientera lui aussi le choix des termes employés. Il se peut en effet que certaines catégories propres à la langue cible soient absentes de la langue source, ou que, pour une catégorie commune, les deux langues aient adopté une terminologie différente. Faut-il en ce cas privilégier la terminologie et le métalangage proposés par les grammaires de FLM, au détriment de la compréhension mais en faveur de la cohérence, ou au contraire recourir à un métalangage et à une terminologie connus des apprenants afin de leur faciliter l'accès à la LE ? Ces questions ont jusque-là fait l'objet de peu de recherches.

Dans le domaine espagnol-français, les travaux portent essentiellement sur la variation terminologique, d'un point de vue diachronique (analyse des dénominations d'une même notion dans plusieurs grammaires du français ou de l'espagnol), ou synchronique et contrastif (comparaison des dénominations d'une même notion dans les grammaires du français et de l'espagnol) ${ }^{2}$. On ne trouve en revanche aucune étude sur la cohérence terminologique au sein d'une même grammaire de FLE à usage d'apprenants hispanophones. Or le problème se pose avec une particulière acuité lorsque ce type de grammaire emploie la méthode contrastive comme procédé de contextualisation. À partir des années 1970, un certain nombre de grammaires de FLE ${ }^{3}$ font en effet du contraste avec la langue maternelle, remis au goût du jour par le structuralisme américain et le développement de la linguistique contrastive au milieu $\mathrm{du} \mathrm{XX} \mathrm{X}^{\mathrm{e}}$ siècle ${ }^{4}$, un élément central de la transposition didactique. La terminologie en est-elle affectée? Quelle terminologie adopter si l'objectif est de concilier qualité du discours grammatical et efficacité didactique ? C'est ce qui va être examiné à présent dans l'ouvrage d'Echeverría, qui fait du contraste avec la langue maternelle des apprenants un élément structurant. 


\section{Présentation du Manual de Gramática francesa et conceptions terminologiques de son auteur}

5 L'espagnole Elena Echeverría Pereda, auteure du Manual de Gramática francesa, enseigne actuellement à la Faculté de Traduction et d'Interprétation de l'Université de Málaga, après avoir enseigné le français dans plusieurs collèges et lycées. Elle fait donc partie des enseignants de langue étrangère qui, confrontés aux difficultés d'apprentissage de leurs élèves, ont éprouvé le besoin d'adapter le discours grammatical existant afin de prévenir ces difficultés ou les corriger plus efficacement. Sachant que les choix terminologiques des grammairiens sont en partie guidés par la théorie linguistique qui préside à l'élaboration de la grammaire, il convient de préciser qu'Echeverría ne se réclame d'aucune théorie linguistique particulière; elle revendique au contraire l'éclectisme et la multidirectionnalité (Echeverría 2011:32), de sorte que l'on peut supposer que ses choix en matière de terminologie s'appuient d'une part sur les éléments transmis par la tradition grammaticale dans les grammaires de référence récentes du français et de l'espagnol, d'autre part sur sa propre expertise. Afin d'évaluer la terminologie du Manual de gramática francesa, nous nous sommes donc référée à des grammaires dont nous supposons qu'elles ont pu être consultées par Echeverría : celles de Grevisse (2011) et de Riegel et al. (1994) pour le français ; celles de la Real Academia Española (1974) et de Bosque \& Demonte (1999) pour l'espagnol. Si la grammaire de la Real Academia Española/Asociación de Academias de la Lengua Española (2010), publiée postérieurement à la première édition du Manual de gramática francesa, n'a pu servir de référence à Echeverría, elle représente néanmoins le point d'aboutissement le plus récent de la tradition grammaticale espagnole, raison pour laquelle elle nous a également servi de référence.

6 Le parcours académique d'Echeverría explique que le Manual de Gramática francesa, publié pour la première fois en 2006 et réédité en 2011 (édition ici utilisée), vise un public hispanophone varié: non seulement des débutants souhaitant passer «le premier niveau du cursus "Langue Française" $»^{5}$ de l'enseignement secondaire ou des Écoles officielles de langues, mais aussi des étudiants de philologie ou de traduction/ interprétation, enfin des apprenants qui souhaitent approfondir leur usage du français et acquérir un niveau supérieur, traducteurs, philologues, professeurs. Cet éventail très large est sans doute ce qui justifie que l'ouvrage ne comporte qu'un seul volume, organisé grosso modo en fonction des "parties du discours ", à l'image des grammaires de consultation. Cette grammaire porte pourtant le nom de "manuel», terme qui l'inscrit dans la lignée des grammaires pédagogiques du deuxième tiers du $\mathrm{XX}^{\mathrm{e}}$ siècle, lesquelles promouvaient une nouvelle forme d'enseignement, plus scolaire, en rupture avec le contenu des grammaires dites «traditionnelles »: il est fort probable qu'en dépit de son organisation traditionnelle, le Manual de gramática francesa soit l'ouvrage qu'utilise Echeverría dans ses cours de traduction.

7 La méthode employée est directement liée au public visé, hispanophone: «nous pensons que le contraste entre la langue objet d'étude et la langue maternelle facilite l'acquisition de la première $»^{6}$. Le Manual de gramática francesa se distingue en effet d'autres grammaires de FLE publiées avant lui et faisant également usage de la méthode contrastive, par le rôle structurant qu'y joue pour la première fois le contraste avec la langue maternelle: l'ouvrage exploite le contraste espagnol-français à différents niveaux visibles dès la table des matières, par de constants va-et-vient entre les deux 
langues, en prenant souvent comme angle d'approche l'espagnol, et non le français. Comment la terminologie trouve-t-elle à s'insérer dans cette logique contrastive?

\section{Catégories grammaticales communes aux deux langues}

8 La langue espagnole et la langue française partagent un grand nombre de catégories ${ }^{7}$ qui ont été nommées de façon similaire par la tradition grammaticale: ils n'ont par conséquent pas posé de problème dans le Manual de gramática francesa. C'est le cas des " articles contractés " ou "élidés ", (artículos contractos), des "structures de mise en relief» ou "emphatiques» (estructuras enfáticas), des "adjectifs démonstratifs" (adjetivos demostrativos) etc. Le cas de l'article, néanmoins, s'est révélé surprenant. Les catégories dénommées en français "article défini » et "article indéfini » existent en espagnol où elles ont reçu deux dénominations dans les grammaires de référence: artículo definido/indefinido ou artículo determinado/indeterminado. L'Esbozo de una nueva gramática de la lengua española (1974) et la Gramática descriptiva de la lengua española (1999) mentionnent les deux dénominations dans l'index alphabétique de notions, mais emploient exclusivement les qualificatifs definido/indefinido dans le chapitre consacré aux articles. Seule la Nueva gramática de la lengua española (2010) emploie préférentiellement le couple determinado/indeterminado, tout en faisant mention des deux dénominations. Bien que la première soit l'équivalent littéral (étymologique) du terme français, c'est la seconde, non employée dans les grammaires françaises, qu'a retenue Echeverría. Étant donné que l'opposition definido/indefinido existe en espagnol, il est regrettable qu'une grammaire du français ait fait le choix de l'autre couple terminologique, absent du français, et moins courant en espagnol.

D'autres catégories communes ont reçu une dénomination différente dans les deux langues, d'où la nécessité de choisir entre la terminologie espagnole et la terminologie française. C'est parfois la terminologie espagnole qui est retenue, tandis que la terminologie française est indiquée entre parenthèses. Le procédé s'observe, de façon très homogène, pour les temps verbaux, ce qui est légitime pour des apprenants débutants : «Formación del Pretérito Perfecto Simple o Pretérito Indefinido (Passé simple) » (Echeverría $2011:$ 150). La grammaire offre un tableau comparatif très clair (Echeverría 2011 : 131) qui met en regard les temps espagnols et les temps français, ce qui permet, selon Avendaño Anguita (2016: 86), de mettre en évidence le parallélisme formel, tout en maintenant la singularité des valeurs attachées à ces temps dans les deux langues.

10 Dans d'autres cas, le résultat manque de cohérence, par exemple dans le chapitre sur les subordonnées. C'est globalement la terminologie espagnole qui est adoptée et, dans une certaine mesure, le Manual de gramática francesa reflète l'absence d'uniformité terminologique des grammaires de référence de l'espagnol. L'Esbozo de una nueva gramática de la lengua española (1974:514) rappelle que le critère retenu pour classer les différentes subordonnées est celui de leur fonction dans la phrase : celles qui ont les mêmes fonctions que le substantif sont nommées oraciones sustantivas (ainsi «que venga », qui a pour fonction sujet du verbe « fastidiar » dans la phrase « me fastidia que venga ", est une oración sustantiva); les subordonnées pouvant avoir dans la phrase les mêmes fonctions que l'adjectif sont nommées oraciones adjetivas (par exemple «que queráis » dans « Coged los cuadernos que queráis »); enfin les subordonnées qui jouent 
dans la phrase le rôle de compléments circonstanciels sont nommées oraciones circunstanciales. L'Esbozo signale néanmoins que les subordonnées « adjetivas » peuvent aussi être appelées «de relativo", en référence au pronom ou à l'adverbe relatif qui les relient à la principale. La gramática descriptiva de la lengua española parle quant à elle de "subordinadas relativas" (Bosque \& Demonte 1999: 3209), et de "subordinadas adverbiales" (Ibid. : 3285) plutôt que de "subordinadas circunstanciales». Enfin, la Nueva gramática de la lengua española emploie les terminologies "subordinadas de relativo" ou "relativas» (RAE 2010: 835), et ne range les subordonnées circonstancielles sous aucune dénomination commune.

11 Au début du chapitre consacré aux subordonnées, Echeverría (2011:305) mentionne les trois catégories existantes au moyen des dénominations espagnoles "sustantivas", «adjetivas » et "adverbiales» (2011: 305$)$, lesquelles s'appuient sur le critère de la fonction de la subordonnée dans la phrase: il y a dans ce cas «modification de l'appellation de la catégorie [...] sans que le contenu qu'elle désigne soit modifié " (Bruley et al. 2012 : 18-19). Les subordonnées «sustantivas » sont en effet, quoique de façon elliptique, définies par leur fonction: Echeverría indique uniquement que les propositions subordonnées substantives peuvent avoir différentes fonctions (sujet, attribut, apposition, complément direct, complément indirect, complément du substantif, complément de l'adjectif, complément de l'adverbe). Un lecteur averti (philologue ou linguiste) sait que ces fonctions sont celles que peut avoir le substantif dans une phrase, et que c'est pour cette raison que ces subordonnées, qui peuvent avoir de telles fonctions, sont nommées «sustantivas » en espagnol. Néanmoins, la majorité des apprenants l'ignorent et la grammaire d'Echeverría ne leur donne pas les moyens de le comprendre. Par ailleurs, pour évoquer, parmi les subordonnées substantives, celles qui jouent le rôle de complément direct du verbe principal, Echeverría utilise une étiquette absente des grammaires de référence et, par ailleurs, très discutable: « sustantivas completivas ». Les subordonnées dites « complétives » correspondent en français à l'ensemble de celles que les grammaires espagnoles nomment "sustantivas ", et non seulement à celles qui jouent le rôle de complément direct du verbe principal. Quant à la section consacrée à la deuxième catégorie de subordonnées, elle s'intitule «oraciones subordinadas adjetivas o de relativo » et ce n'est pas le critère de la fonction de la subordonnée mais bien celui, français, du terme introducteur ${ }^{8}$, qui sert à

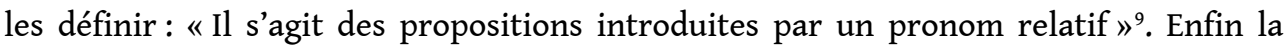
section consacrée aux dernières porte un titre, « oraciones subordinadas adverbiales $o$ circunstanciales ", qui a le mérite d'évoquer la terminologie française (« subordonnées circonstancielles ») mais qui n'est assorti d'aucune définition.

Pour les catégories grammaticales communes aux deux langues, le choix global de la terminologie espagnole est parfaitement légitime si l'objectif est de faciliter la tâche d'apprentissage. La grammaire d'Echeverría s'adresse en effet à des apprenants, du secondaire ou au-delà, qui ont acquis une certaine culture métalinguistique ; le système des subordonnées et de leur classement est l'un des points complexes de la grammaire (tant espagnole que française) et contraindre les apprenants à se familiariser avec une autre terminologie reviendrait à leur compliquer inutilement la tâche. On regrette néanmoins l'absence de définitions claires et toujours cohérentes avec la terminologie et la présence d'une étiquette erronée susceptible de désorienter les apprenants qui utiliseraient d'autres grammaires privilégiant la terminologie française. 


\section{Catégories dissemblables ayant reçu des dénominations similaires}

13 Observons à présent le cas des catégories qui ont reçu des dénominations très similaires en français et en espagnol, mais qui recouvrent des réalités différentes. Choisir la terminologie espagnole, c'est alors employer une appellation qui ne correspond pas au contenu décrit, comme le fait Echeverría (2011) lorsqu'elle emploie les dénominations de «complemento directo $(C D)$ » et «complemento indirecto (Cind)»; ces catégories espagnoles n'ayant pas reçu la même définition que les catégories dénommées dans la grammaire française "complément d'objet direct (COD)» / « complément d'objet indirect (COI)», elles n'englobent pas exactement les mêmes types de compléments (Pino Serrano \& Valcárcel Riveiro 2016: 21). Les définitions françaises, à la fois sémantiques et formelles, reposent sur la notion d'« objet ", et sur l'idée de construction directe, sans préposition, de cet objet :

Dans la phrase canonique de base, les verbes transitifs directs sont normalement suivis d'un complément d'objet (abrégé en c.o.d) construit sans préposition et qui, sauf blocage sémantique, peut prendre toutes les formes du groupe nominal et de ses équivalents pronominaux ou prépositionnels [...] Le c.o.d prototypique est un second actant qui, sémantiquement, joue le rôle de terme final du processus relationnel instauré par le verbe: objet affecté (Il visite la maison), effectué (Il construit la maison), etc. (Riegel et al. 1994 : 221-222)

Par opposition, le « complément d'objet indirect » est celui dont l'objet est construit indirectement, avec préposition :

Le complément d'objet direct (ou simplement objet direct) est rattaché au verbe directement, c'est-à-dire par l'intermédiaire d'une préposition. (Grevisse 2001 : 394)

Une telle définition implique que sous la catégorie "complément d'objet indirect » soient en réalité rangées plusieurs sous-catégories : le complément d'objet indirect au sens strict, complément unique de certains verbes construits avec préposition (« Penser à son malheur ", "Parler de politique ", "Habiter à Paris»), mais également le complément d'attribution, qui, selon la tradition grammaticale ${ }^{10}$, désigne plus particulièrement le second complément des verbes à deux compléments ( «Le professeur donne une récompense à Paul »). En espagnol, en revanche, le $C D$ est défini et caractérisé par la transitivité, la passivation et la pronominalisation. Le Cind, quant à lui, renvoie à la fonction dative qui correspond, formellement, à des groupes prépositionnels introduits uniquement par la préposition «a » et pronominalisables en «le ». Un certain nombre des COI de la grammaire française n'entrent donc pas dans la catégorie Cind de la grammaire espagnole ${ }^{11}$.

Le choix de la terminologie espagnole dans le Manual de gramática francesa aura sans doute un effet rassurant auprès d'apprenants débutants ainsi ramenés à du connu ; elle risque en revanche de poser des problèmes de cohérence voire de compréhension à des apprenants plus avancés.

Un autre exemple de ce phénomène nous est donné dans la section «Diminutivos y aumentativos» (Echeverría 2011: 99) du chapitre 3 consacré au nom. La norme est formulée de la façon suivante :

Para formar aumentativos, en francés se utilizan los adjetivos Grand (estatura) y

Gros (tamaño, exceso) [...]

Para formar diminutivos, en francés se utiliza el adjetivo Petit delante del nombre ${ }^{12}$.

(Echeverría 2011 : 99) 
La formulation est ambiguë en raison d'une non correspondance entre les deux langues: alors que les termes "augmentatif»/aumentativo font référence non seulement à un élément de formation (suffixe), mais également au mot affecté de cet élément ${ }^{13}$, le terme "diminutif » ne fait référence en français qu'à un élément de formation, contrairement à son correspondant espagnol ${ }^{14}$. Or c'est bien dans le sens de " mot affecté d'un tel élément », sens le moins usuel dans les grammaires françaises et espagnoles de référence, qu'Echeverría emploie ici les termes "diminutivo " et "aumentativo». Ce choix permet de ranger sous une même dénomination les syntagmes "adjectif + nom » du français et les substantifs de discours obtenus par l'ajout de suffixes diminutifs ou augmentatifs de l'espagnol et ainsi de les évoquer de façon contrastive sans avoir recours à une catégorie propre à la langue source. La cohérence théorique de l'exposé est néanmoins discutable: si l'exposé de la première partie de la norme fonctionne (les appellations "augmentatif» et "aumentativo" renvoyant aux mêmes catégories), la seconde partie manque de rigueur puisque l'appellation « diminutivo » ne recoupe pas exactement la même catégorie que celle de « diminutif »; il y a donc ici modification du contenu d'une catégorie, sans modification de l'appellation. Du point de vue de l'efficacité didactique, ces termes risquent par conséquent d'engendrer des confusions chez les apprenants.

\section{Catégories propres à la langue française}

Concernant ces catégories qui n'ont pas d'équivalent en espagnol, une première solution, dans le Manual de gramática francesa, est la traduction ; c'est ce qui a été réalisé pour la catégorie de l'«article partitif» qui n'existe pas en espagnol:«artículo partitivo " (Echeverría 2011: 80). On peut imaginer que cette solution est adoptée lorsqu'une traduction est possible et «transparente » : l'adjectif "partitif » est dérivé du substantif "partie» qui se dit parte en espagnol, en vertu d'un étymon latin commun (PARS, -TIS, f.) ; on peut donc construire sans difficulté l'adjectif partitivo en espagnol.

Une autre solution est le maintien du terme français en italiques. Les concepts d'«élision" et de "liaison", par exemple, qui renvoient à des phénomènes phonétiques absents de l'espagnol, sont maintenus tels quels (Echeverría 2011:67) et définis dans l'annexe 2 (Echeverría 2011 : 357-358) consacrée à la phonétique. En effet, le terme elisión existe en espagnol, mais ne renvoie pas au même phénomène que le terme français. En français, l'élision est un type d'apocope consistant en l'amuïssement de la voyelle finale d'un mot devant un autre mot à initiale vocalique; elle est marquée graphiquement par une apostrophe (ex: «l'arbre»). En espagnol, le phénomène ne concerne que l'amuïssement de la voyelle initiale des articles contractés (Ex : $a+e l \rightarrow$ al). Il est donc légitime de n'avoir pas traduit ce terme. Quant à la «liaison", prononciation de la consonne finale d'un mot devant un autre qui commence par une voyelle, c'est un phénomène qui n'existe pas en espagnol, et le terme ne peut être traduit par un équivalent étymologiquement proche (le plus proche serait relación ou conexión), raison pour laquelle il a été maintenu en français. 


\section{Catégories propres à la langue espagnole}

L'originalité de l'ouvrage d'Echeverría consiste enfin en l'introduction, dans une grammaire du français, de catégories propres à la langue espagnole. Il ne s'agit pas de l'introduction de simples dénominations propres à la langue espagnole renvoyant à des phénomènes présents dans les deux langues, mais bien de catégories ou de phénomènes propres à la grammaire espagnole, et n'ayant pas de correspondant dans la grammaire française. On évoquera par exemple les «Incrementos átonos de interés " (Echeverría 2011 : 69), constructions dites pseudo-réfléchies dans la mesure où elles n'ont pas un sens réfléchi ou réciproque et où, par conséquent, le morphème se n'est pas un pronom mais un clitique. Il est ici question plus particulièrement de ce que les grammaires nomment «datif d'intérêt» ou "datifs superflus» (Gutiérrez Ordóñez 1999: 1910), constructions dans lesquelles la présence du clitique se, facultative, est associée à un signifié aspectuel ${ }^{15}$ : « Después de comer, me tomé un café » (Echeverría $2011: 70$ ), « Me leí dos capítulos » (Echeverría ibid.). Echeverría consacre à ce phénomène une section du chapitre consacré aux articles; elle aborde en effet, selon une description contrastive inverse, cette construction typique de la langue espagnole qui consiste à faire suivre un verbe pronominal d'un article pour indiquer un rapport de possession : "Quítate la corbata », «A los 15 años, se ganaba ya la vida » (Echeverría 2011 : 69). Il s'agit de prévenir des incorrections dues à un calque de l'espagnol: «enlève-toi la cravate» ou «À 15 ans, elle se gagnait déjà la vie». La section suivante, intitulée « Diferencia español-francés : incrementos átonos de interés ", constitue une sorte de synthèse de ce paragraphe; la catégorie des incrementos átonos de interés, inexistante en français, n'est pas ici importée (la dénomination ne sert pas à décrire une catégorie de la grammaire française) mais se trouve intégrée à l'ouvrage comme si elle appartenait à la grammaire française. Pour une explication du mécanisme de ces constructions, l'auteure renvoie à un ouvrage du linguiste espagnol Alarcos Llorach, se contente de signaler qu'« elles n'ont généralement pas de correspondance formelle en français $»^{16}$ (Echeverría ibid.) et indique la construction équivalente en français.

De la même façon, le Manual de gramática francesa évoque les phénomènes de leísmo, loísmo et laísmo qui ne renvoient pas à proprement parler à des catégories mais à des phénomènes de substitutions de pronoms propres à l'espagnol: respectivement l'emploi des pronoms le/les à la place de lo/los pour la fonction d'objet direct, l'emploi de lo/los à la place de le/les pour la fonction objet indirect, enfin l'emploi de la/las à la place de le/les pour la fonction objet indirect (référent féminin). Ces phénomènes, totalement absents de la langue française, constituent le titre à part entière de l'une des sections du chapitre 6 consacré notamment aux pronoms personnels; ce titre annonce d'emblée l'absence de correspondance entre les deux langues: "Leísmo, laísmo y loísmo : fenómenos inexistentes en francés » (Echeverría 2011 : 197).

\section{Conclusion}

Publié par une maison d'édition espagnole, le Manual de gramática francesa pourrait être qualifié de "grammaire espagnole du français pour hispanophones » : les choix faits en matière de terminologie s'inscrivent pleinement dans cette optique et dans le parti pris contrastif qui structure l'ouvrage, en adéquation avec le public visé (étudiants en traduction). Pour les catégories grammaticales communes aux deux langues, c'est 
globalement la terminologie espagnole qui est employée, vraisemblablement dans un souci d'efficacité didactique : l'apprenant, ramené à du connu, abordera l'apprentissage avec plus de confiance. Encore faut-il que ce principe soit mis en œuvre de façon rigoureuse, ce qui n'est pas toujours le cas chez Echeverría : en dehors du chapitre sur les temps verbaux qui, avec une grande rigueur, fait usage de la terminologie espagnole, tout en indiquant systématiquement la terminologie française entre parenthèses, ailleurs le mélange des deux terminologies et l'absence de définitions claires et cohérentes avec les dénominations employées risquent de susciter interrogations voire difficultés chez les apprenants.

C'est aussi la terminologie espagnole qui est majoritairement employée même si les catégories ne se recoupent que partiellement entre les deux langues, d'où un certain nombre d'incohérences théoriques susceptibles d'être sources de confusions chez des apprenants ayant déjà une bonne culture métalinguistique espagnole. D'autres grammaires de FLE pour hispanophones employant la perspective contrastive se montrent, de ce point de vue, plus cohérentes; on pense, parmi les grammaires qui utilisent l'espagnol comme langue véhiculaire, à celle de Capelle et al. (1981) ou, plus récemment, à celle de Di Giura \& Suso López (2013 et 2015) ou à celle de Benítez López (2020) qui, bien que rédigée en espagnol, emploie exclusivement la terminologie française, y compris pour les temps verbaux. Resterait à comparer la façon dont les unes et les autres sont effectivement reçues par les apprenants hispanophones, mais aussi, éventuellement, à comparer la première édition (2006) du Manual de gramática francesa avec l'édition consultée ici (2011), laquelle ne consiste en effet pas en une simple réimpression de la première: Echeverría a-t-elle introduit des modifications terminologiques justifiées par le feed-back avec ses étudiants? Les dénominations de catégories n'ayant pas d'existence dans la langue française, élément sans doute le plus original du Manual de gramática francesa, étaient-elles déjà présentes dans l'édition initiale? Autant de passionnantes questions qui, nous l'espérons, ouvriront la voie à de nouvelles recherches.

\section{BIBLIOGRAPHIE}

ALARCOS LLORACH, Emilio (1994 [1970]). Estudios de gramática funcional del español. Madrid : Gredos. CNRTL (2002). Trésor de la langue française informatisé. En ligne : [http://atilf.atilf.fr/tlfv3.htm $(16 / 02 / 2021]$.

AVENDAÑO ANGUITA, Lina (2016). « Le verbe français : les temps du passé ». Synergies Espagne, 9, 83-93.

BEACCO, Jean-Claude (2015). « Mouvements terminologiques et contextes. Introduction à la problématique ». In Cécile Bruley \& Javier Suso López (coords.). La terminología gramatical del español y del francés : emergencias y transposiciones, traducciones y contextualizaciones // La terminologie grammaticale de l'espagnol et du français : émergences et transpositions, traductions et contextualisations. Francfurt : Peter Lang, 11-24. 
BENÍTEZ FLORES, Carlos Y. (2020). Gramática francesa básica para hispanohablantes : La francophonie et moi. Format Kindle.

BOSQUE, Ignacio \& DEMONTE, Violeta (1999). Gramática descriptiva de la lengua española. Madrid : Real Academia Española / Espasa Calpe.

BRULEY, Cécile et al. (2012). "Grammaires du français et discours grammaticaux contextualisés ». In José Ignacio Aguilar Río, Cédric Brudermann \& Malory Leclère (dir.). Complexité, diversité et spécificité : Pratiques didactiques en contexte. Paris : Presses Sorbonne Nouvelle, 10-23.

BRULEY, Cécile \& SUSO LÓPEZ, Javier (coords.) (2015). La terminología gramatical del español y del francés: emergencias y transposiciones, traducciones y contextualizaciones // La terminologie grammaticale de l'espagnol et du français : émergences et transpositions, traductions et contextualisations. Frankfurt : Peter Lang.

CANTERA ORTIZ DE URBINA, Jesús \& DE VICENTE, Eugenio (1974). La Gramática francesa en cuadros esquemáticos. Madrid : E.P.E.S.A.

CAPELLE, Guy. et al. (1994). Gramática básica de la lengua francesa. Paris : Hachette.

CHEVALLARD, Yves (1991). La transposition didactique. Du savoir savant au savoir enseigne - Grenoble : La pensée sauvage éditions.

DULIN BONDUE, Nicole, PALAFOX, Sylvie \& OZAETA GALVEZ, Ma Rosario (2011). L'essentiel Français Langue Étrangère. Madrid : UNED.

ECHEVERRÍA PEREDA, Elena (2011 [2006]). Manual de gramática francesa. Barcelona : Ariel Letras.

FEUILLET, Jack (1986). « Catégories et fonctions ». L'information grammaticale, 31, 3-7.

FERNÁNDEZ-BALLÓN, Manuel \& MONNERIE-GOARIN, Annie (1987). Gramática esencial de francés. Paris :

Larousse.

FOUILLET, Raphaële (2014). « Les 'recettes contrastives' dans les grammaires du français pour italophones ». Langue française, 181, 19-36.

FOURNIER, Jean-Marie (2013). Histoire des théories du temps dans les grammaires françaises. Lyon : ENS Éditions.

FRIES, Charles C. (1945). Teaching and Learning English as a Foreign Language. Ann Arbor : University of Michigan Press.

GIURA (di), Marcella \& SUSO LÓPEZ, Javier (2013). Grammaire contrastive para hispanohablantes A1/A2. Paris : Nathan-CLE international.

GIURA (di), Marcella \& SUSO LÓPEZ, Javier (2015). Grammaire contrastive para hispanohablantes B1/B2. Paris : Nathan-CLE international.

GÓMEZ ASENCIO, José J. (2011). Los principios de las gramáticas académicas (1771-1962). Bern, Berlin, Bruxelles etc. : Peter Lang.

GREVISSE, Maurice (2001 [1986]). Le bon usage. Edition par André Goosse. Paris : Duculot.

GUTIÉRREZ ORDóÑEZ, Salvador (1999). « Los dativos ». In Ignacio Bosque \& Violeta Demonte (dir.). Gramática descriptiva de la lengua española, vol. 2. Madrid : Real Academia Española / Espasa Calpe, 1855-1930.

HASSLER, Gerda (2014). « Los tiempos verbales y sus denominaciones en las gramáticas españolas desde el siglo XVII hasta el siglo XX ». In María Luisa Calero et al. (eds.). Métodos y resultados actuales en Historiografía de la Lingüística. Münster : Nodus-Publikationen, 349-360. 
JOB, Beatriz (2013). La grammaire. Français : théorie et pratique. Madrid : Santillana.

LADO, Robert (1957). Linguistics accross culture : applied linguistics for language teachers. Ann Arbor : University of Michigan Press.

MARTÍNEZ GARCíA, Hortensia (1986). El suplemento en español, Prólogo de E. Alarcos Llorach. Madrid : Gredos.

MERRILEES, Brian (1988). « Les débuts de la terminologie grammaticale en français : à propos de quelques travaux récents ». Romania, 109, 435/435, 397-411.

PINO SERANO, Laura et VALCÁRCEL RIVEIRO, Carlos (2016). « Los complementos del verbo en francés y su contextualización en las gramáticas locales españolas ». Synergies Espagne, 9, 17-33.

QUIJADA VAN DEN BERGHE, Carmen \& FOURNIER, Jean-Marie (2017). « La descripción de los pretéritos perfectos en las gramáticas españolas del foco francés (1596-1800) ». Moenia : Revista lucense de lingüistica \& literatura, 23, 179-219.

QUIJADA VAN DEN BERGHE, Carmen (2019). « Categorización verbal de las referencias al pasado en gramáticas escolares de primera mitad del siglo XX ». In María José García Folgado, Carsten Sinner \& Guillermo Toscano (eds.). Clases y categorías en la gramática española desde una perspectiva historiográfica. Leipzig : Leipziger Universitätsverlag, 91-114.

REAL ACADEMIA ESPAÑola (RAE) (1974 [1973]). Esbozo de una nueva gramática de la lengua española. Madrid : Espasa.

REAL ACADEMIA ESPAÑola (RAE) (1998 [1992]). Diccionario de la lengua española. Madrid : Espasa Calpe. REAL ACADEMIA ESPAÑOLA (RAE)/ASOCIACIÓN DE ACADEMIAS DE LA LENGUA ESPAÑOLA (2010 [2009]). Nueva gramática de la lengua española. Madrid : Espasa Libros.

RIEGEL, Martin, PELLAT, Jean-Christophe \& RIOUL, René (1994). Grammaire méthodique du français. Paris : PUF.

\section{NOTES}

1. On mentionnera, pour l'espagnol, Gómez Asencio (2011), Haßler (2014), Quijada Van den Berghe \& Fournier (2017). Pour le français, on se reportera notamment à Merrilees (1988) et Fournier (2013).

2. Ce sont en effet les différents aspects qu'abordent les travaux regroupés par Bruley \& Suso López (2015) ou l'article de Quijada Van den Berghe (2019).

3. On citera, par ordre chronologique de publication, les grammaires de Cantera \& De Vicente (1986), de Fernández-Ballón \& Monnerie-Goarin (1987), de Capelle (1994), de Echeverría Pereda (2006), de Dulin Bondue et al. ([Première édition 2006], 2011), de Job (2013), de di Giura \& Suso López (2013 et 2015) et de Benítez Flores (2020).

4. Au fondement de ce courant, on citera en particulier les réflexions de Fries (1945) et de Lado (1957).

5. « nivel inicial de la asignatura denominada "Lengua Francesa"» (Echeverría 2011 : 31).

6. «creemos que el contraste entre la lengua objeto de estudio y la lengua materna facilita la adquisición de la primera » (Echeverría Pereda $2011: 31$ ).

7. On entendra ici le terme de "catégorie » au sens large, c'est-à-dire non seulement les traditionnelles "parties du discours» (noms, verbes, adjectifs, déterminants etc.) mises en évidence dans l'Antiquité grecque puis latine, mais aussi les morphèmes grammaticaux entrant dans la composition du mot (désinences, flexions, affixes), enfin les fonctions grammaticales 
(COD, COI, complément circonstanciel etc.), souvent confondues par les linguistes avec les parties $\mathrm{du}$ discours. Sur ce dernier point, voir Feuillet (1986:2-3).

8. En français, le critère retenu est celui du mot introducteur de la subordonnée: les subordonnées introduites pas un pronom relatif sont appelées «subordonnées relatives »; celles introduites pas une conjonction de coordination sont appelées « subordonnées conjonctives », et celles-ci se subdivisent en « subordonnées essentielles » ou « complétives » si elles apportent un complément d'information essentiel, et « «subordonnées circonstancielles» si elles indiquent une circonstance de l'action non essentielle.

9. «Se trata de las oraciones introducidas por un pronombre relativo » (Echeverría $2011: 310$ ).

10. C'est ce qu'indiquent Riegel et al.: «La tradition grammaticale appelle complément d'attribution l'objet second introduit par à, bien que ce rapport ne caractérise pas tous les objets seconds construits avec cette préposition (certains comme oter, confisquer, arracher etc. sont même l'objet du processus inverse de dépossession) » (1994 : 225-226).

11. Alarcos Llorach (1994: 156-157) nomme suplemento un complément tel que «de política» dans « hablar de política " (parler de politique) et suplemento inherente le complément " en París » dans « Residir en Paris » (Vivre à Paris) (Martínez García 1986 : 4). Pour ces derniers, les manuels Lengua española utilisés dans le secondaire, de même que les différentes éditions de la grammaire de la Real Academia española $(1999,2009,2010)$ emploient la dénomination « complemento de regimen preposicional » (Pino Serrano \& Valcárcel Riveiro 2016 : 32).

12. «Pour former des augmentatifs, en français on emploie les adjectifs Grand (taille) et Gros (embonpoint, excès) [...] Pour former des diminutifs, en français on emploie l'adjectif Petit devant le nom. » (Notre traduction)

13. "Augmentatif » : "Élément de formation ou adverbe, qui contribue à donner aux mots une signification superlative plus énergique. P. ext. Le mot affecté d'un tel élément » (CNRTL 2002:s. v. "augmentatif »). Aumentativo: «2. adj. Gram. Dicho de un sufijo: Que expresa aumento, intensidad o exceso de lo denotado por el vocablo al que se une »; «3. m. Gram. Palabra formada por uno o más sufijos aumentativos " (RAE 1998 : s. v. aumentativo).

14. « Diminutif » : « Mot ou élément de formation (d'ordinaire suffixe) qui convient à l'expression de la petitesse » (CNRTL 2002 : s.v. « diminutif »); Diminutivo : «2. Adj. Gram. Dicho de un sufijo : que expresa disminución, atenuación o intensidad de lo denotado por el vocablo al que se une, o que valora afectivamente su significación »; «3. m. Gram. Palabra formada con uno o más sufijos diminutivos » (RAE 1998 : s. v. diminutivo).

15. Parmi ces valeurs aspectuelles, on notera « la gradualidad de la acción, la consumación de la totalidad del proceso (valor perfectivo), el carácter cuantificado del complemento directo etc. » (Gutiérrez Ordóñez 1999 : 1911). [Notre traduction : «la gradualité de l'action, la consommation de la totalité du procès (valeur perfective), le caractère quantifié du complément direct etc. »] ou encore l'idée d'un fait qui va à l'encontre des attentes (Gutiérrez Ordoñez 1999 : 1915).

16. « carecen por lo general de correspondencia formal en francés».

\section{RÉSUMÉS}

Cet article s'interroge sur la terminologie employée dans le Manual de gramática francesa de Echeverría Pereda (2006), grammaire pédagogique du français langue étrangère destinée à des apprenants hispanophones. Les travaux portant sur le problème de la terminologie dans les 
grammaires de FLE se sont peu intéressés à la cohérence terminologique au sein d'une même grammaire, problème majeur si l'on prend en compte qu'à partir des années 1970, de nombreuses grammaires de FLE, et tout particulièrement celle de Echeverría Pereda, réintroduisent la perspective contrastive avec la langue maternelle comme méthode d'apprentissage. Comment la terminologie trouve-t-elle à s'insérer dans la logique contrastive ? Comment concilier cohérence théorique et efficacité didactique ? Par l'analyse de différents cas de figure, on montrera que si les choix faits par Echeverría en matière de terminologie s'inscrivent dans le parti pris contrastif qui structure l'ouvrage et peuvent se révéler efficaces dans certains cas, ils souffrent dans d'autres cas d'un manque de cohérence et de rigueur qui risque de faire obstacle à l'apprentissage.

This paper examines the terminology used in Elena Echeverría Pereda's Manual de gramática francesa (2006), a pedagogical grammar of French as a foreign language for Spanish-speaking learners. The problem of terminology in FLE grammars has received little attention in the literature, and this is a major problem when one considers that, since the 1970s, many FLE grammars, especially Echeverría Pereda's, have reintroduced the contrastive perspective with the mother tongue as a learning method. How does terminology fit into the contrastive logic? How can one reconcile theoretical coherence and didactic efficiency? By analyzing different cases, we will demonstrate that if the terminological choices made by Echeverría are consistent with the contrastive approach that guides the book and can be effective in some cases, those choices entail, in other cases, a lack of coherence and rigor that may impede learning.

\section{INDEX}

Keywords : French as a foreign language, pedagogical grammars, Manual de gramática francesa, Spanish-speaking learners, terminology, contrastivity

Mots-clés : FLE, grammaires pédagogiques, Manual de gramática francesa, apprenants hispanophones, terminologie, contrastivité

\section{AUTEUR}

\section{ÉLODIE WEBER}

Sorbonne Université - el.web@free.fr 